\title{
Desarrollo de un Analizador en línea, de las concentraciones de Cobre en soluciones acuosas del proceso de Extracción por Solvente, mediante procesamiento de imágenes
}

\author{
Claudio Ayala B. ${ }^{1}$, José Gallardo A. ${ }^{2}$, Manuel Olivares A. ${ }^{3}$ \\ claudio.ayala@uantof.cl,jgallardo@ucn.cl,manuel.olivares@ucn.cl \\ ${ }^{1}$ Departamento de Ingeniería Eléctrica, Universidad de Antofagasta, Av. Angamos 601, 1240000, \\ Antofagasta, Chile. \\ 2,3 Departamento de Ingeniería de Sistemas y Computación, Universidad Católica del Norte, Av. Angamos \\ 0610, 1240000, Antofagasta, Chile.
}

DOI: 10.17013/risti.24.1-12

\begin{abstract}
Resumen: Este artículo presenta el desarrollo de un nuevo analizador, instrumento para medir en línea concentraciones de cobre en acuoso. Su principio de funcionamiento se basa en la aplicación de técnicas de procesamiento de imágenes y un sistema automatizado para la toma de muestras desde el proceso. En principio, este analizador ha sido desarrollado para el proceso de extracción por solvente, que es una de las etapas del proceso hidrometalúrgico para la obtención de cátodos de cobre. Los resultados logrados son alentadores pues el instrumento desarrollado presenta una precisión aceptable para este tipo de instrumentos no convencionales, augurándose mediciones con mayor precisión cuando se implemente el instrumento en su versión industrial, con componentes de mayor calidad y robustez. Con este nuevo instrumento, las empresas mineras tendrán una alternativa de medición en línea de esta importante variable operacional, mejorando la operación de la planta y el incremento en su eficiencia y recuperación.
\end{abstract}

Palabras clave: Analizador, concentración, cobre, extracción, solvente.

\section{Development of a copper concentration on-line Analyzer, for aqueous solutions from the Solvent Extraction process, through images processing and colorimetry}

\begin{abstract}
This article proposes a new analyzer, instrument to measure on line copper concentrations in aqueous. Its operating principle is based on technics of images processing and colorimetry, and an automated system for the taking of samples directly withdrawn from the process. In principle, this analyzer has been developed for the solvent extraction process, which is one of the stages of the hydrometallurgical process to obtain copper cathodes. The results of this development are promising due to the analyzer presents an acceptable precision for this type of non conventional instruments, remaining even a space for improvements
\end{abstract}


when using components in its industrial version, of greater quality and robustness. With this new analyzer, the mining companies will have an alternative to measure on line this important operational variable, improving, with its application, the operation of the plant and increasing its efficiency and recovery.

Keywords: Analyzer, concentration, copper, extraction, solvent.

\section{Introducción}

El proceso hidrometalurgia del cobre, compuesto por las etapas de Lixiviación (LIX), Extracción por Solvente (SX) y Electro-obtención (EW) es uno de los más importantes métodos de producción de cobre desde minerales oxidados de baja ley (Komulainen, Doyle III, Rantala, \& Jämsä-Jounela, 2009). La investigación en este proceso, ha sido enfocada principalmente en los procesos químicos y equipos asociados. Hasta el presente, el control de los procesos de SX-EW ha recaído sobre simples lazos de control y sintonía manual, con operadores manipulando activamente los set-point (SP) para controladores de nivel básico o de primer nivel (Komulainen et al., 2009; Bergha, Jämsä-Jounela, \& Hodouinc, 2001). Un factor clave que reduce la eficiencia de plantas de SX es la metodología reactiva que los operadores de estas plantas están obligados a realizar, basados en análisis de laboratorio de los flujos fuera de línea, los cuales ya han sido procesados a través de la planta (Li, Yuan, Yang, \& Huang, 2007). Los ingenieros de proceso utilizan estos análisis After-the-fact para predecir los mejores parámetros de operación para el subsecuente período de operación. Estos parámetros de operación predichos resultan en ajustes manuales para los parámetros del proceso, los cuales, si son correctos, facilitan una extracción más alta de cobre y rechazo más alto a las impurezas. El nivel de éxito de estas decisiones está sujeto a cómo el staff operacional interpreta el estado de la planta en un momento dado (Li et al., 2007).

Una de las razones de esta forma de operar plantas de SX es la falta de medidores de las variables operacionales más relevantes del proceso, como ser la Razón orgánico-acuoso (O/A), Tiempo de Separación de Fases (TSF) y Concentraciones de Cobre. Sin duda que la variable operacional más importante dentro del proceso de SX es la concentración de cobre en los flujos de salida de las distintas etapas del proceso, principalmente la concentración de cobre del electrolito rico, que sirve de entrada al proceso de electroobtención. Existe por lo tanto la necesidad de medir las concentraciones de cobre en soluciones acuosas en tiempo real, con el objetivo de realizar el control de esta importante variable operacional, en plantas de extracción por solvente.

El principal objetivo de control de una planta de extracción por solvente de cobre es maximizar la recuperación y proveer electrolito rico estable para el proceso de electroobtención (EW) (Komulainen et al., 2009). Una concentración adecuada de cobre en el electrolito rico permite una operación más estable y una operación más eficiente en la obtención de los cátodos de cobre, por estos motivos es muy importante mantener estable su valor operacional lo más cercano posible a las consignas establecidas por el proceso.

Sin embargo, la mayoría de las plantas de SX en Chile y en el mundo, no poseen o disponen de un medidor de concentraciones en línea, principalmente por el elevado costo de estos instrumentos. La mayoría de las plantas realizan medidas de concentraciones fuera de 
línea, sobre muestras tomadas en distintos puntos del proceso, demorando dos o más horas en realizar un análisis de ella en un laboratorio.

Respecto a las alternativas existentes en el mercado de la instrumentación analítica, de medidores de concentración de cobre en línea para procesos mineros, solo existen los analizadores Courier. Este tipo de analizador, permite un muestreo y análisis en forma automática y consistente. La precisión de las mediciones es comparable a la de un laboratorio que analiza muestras manuales de alta calidad. Los analizadores Courier en línea utilizan la misma tecnología de análisis de Fluorescencia de Rayos X por Dispersión de Longitud de Onda (Martínez, Gil Novoa, \& Barón González, 2006), que se utiliza en los analizadores de laboratorio de alto rendimiento. Sus principales desventajas son el alto costo y la necesidad de re-calibración permanente.

En este reporte, se presenta el desarrollo de un nuevo medidor de concentraciones de cobre en línea, basado en el procesamiento de imágenes y la técnica de colorimetría. Algoritmos genéticos son utilizados para la obtención de los parámetros que permitan la mejor estimación del modelo de correlación planteado, es decir, la estimación con el menor error cuadrático, respecto de un universo de muestras con diferentes valores de concentraciones de cobre obtenidas en laboratorio. Con posterioridad, este modelo de correlación es aplicado sobre muestras capturadas en línea directamente del proceso. La principal ventaja de este analizador en línea respecto a lo existente, es su relativo bajo costo.

Este artículo es estructurado como sigue: en la sesión 2 se describe el proceso de mezcla y decantación de la técnica de extracción por solventes y en la sesión 3 un esquema general del instrumento, con una descripción de cada una de sus partes y el principio de medición utilizado. En la sesión 4 de muestran los resultados experimentales y en las sesiones 5 y 6 se presentan las conclusiones, trabajo futuro y referencias bibliográficas respectivamente.

\section{Proceso}

En la Figura 1, se representa un estanque mezclador-decantador, típico de los procesos de extracción y re-extracción utilizados en plantas hidrometalurgias para la obtención de cátodos de cobre.

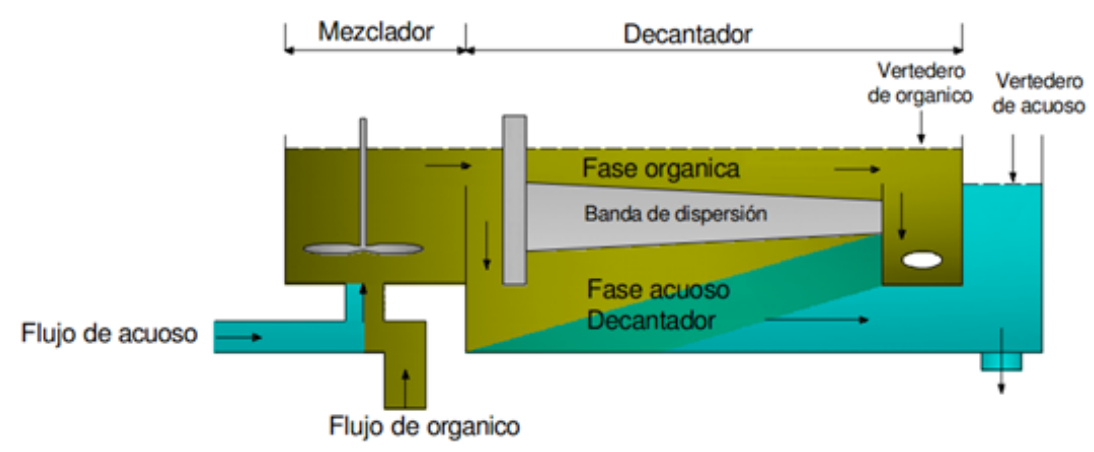

Figura 1 - Estanque mezclador- decantador para el proceso de extracción y re-extracción. 
El orgánico y el acuoso que entran a los estanques principal y secundario son mezclados, produciéndose, durante esta acción, un traspaso de cobre del acuoso al orgánico (o de orgánico a acuoso en re-extracción). La medición se debe realizar en el vertedero de acuoso (salida de acuoso), para conocer la concentración de cobre producto del proceso de extracción (o re-extracción) que se ha producido en esta etapa.

\section{Principio de medición y tecnología implementada}

La tecnología propuesta se basa en la técnica de la colorimetría (Artigas, Capilla, Malo, Martínez, Luque, Pujol, 2002) aplicada a una muestra tomada desde el proceso, utilizando un algoritmo de procesamiento de imágenes. La toma de la muestra y su procesamiento es automatizado para la medición en línea, utilizando dispositivos como válvulas, sensores y un controlador lógico programable.

\subsection{Esquema de medición}

La Figura 2 representa un esquema del sistema para la medición en línea de la concentración de cobre.

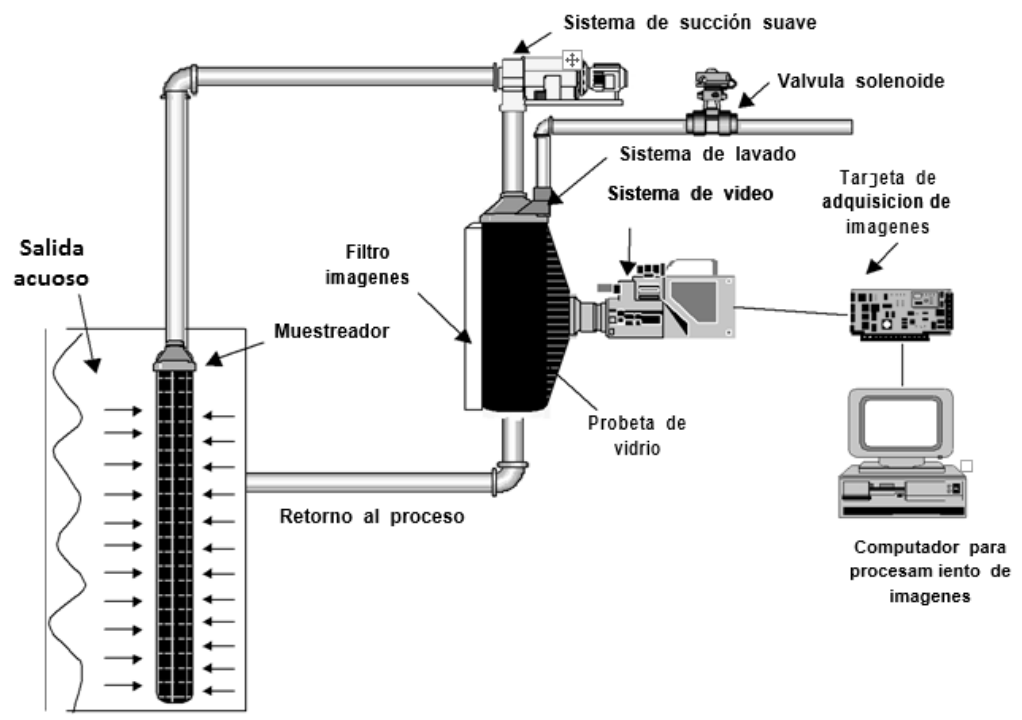

Figura 2 - Esquema del instrumento de medición

Mediante una bomba de succión, se extrae la muestra de acuoso en la salida de cada decantador, donde se desea obtener una medida de la concentración de cobre. Esta muestra se deposita en una probeta de vidrio, previamente sometida a un proceso de lavado, con agua controlada por la válvula solenoide. Luego en forma sincronizada, se toman imágenes de la muestra mediante una cámara y se procesa la información en un computador. Esta secuencia se vuelve a repetir periódicamente en un tiempo que puede ser fijado por el usuario. 


\subsection{Rocesamiento de las imágenes de las muestras}

El proceso de estimación de la concentración de cobre en acuoso se realiza utilizando un simple algoritmo de tratamiento de las tomas (imágenes) captadas de las muestras que se extraen del acuoso. Estas imágenes son descompuestas en sus colores primarios Red, Green y Blues, modelo RGB (Rojo, Verde y Azul). Estos colores primarios, combinados de forma adecuada, conforman una gama amplia de colores, como por ejemplo las combinaciones que se ilustran en la Figura 3.

RGB es un modelo de color basado en la síntesis aditiva, con el cual es posible representar un color mediante la mezcla por adición de los tres colores de luz primarios ( $\mathrm{Li}$ et al., 2007; Sankaranarayanan \& Sivanantha, 2006). El modelo de color RGB, no define por sí mismo lo que significa exactamente rojo, verde o azul, por lo que los mismos valores RGB pueden mostrar colores notablemente diferentes en varios dispositivos que usen este modelo de color, y aunque utilicen un mismo modelo de color, sus espacios de color pueden variar considerablemente.

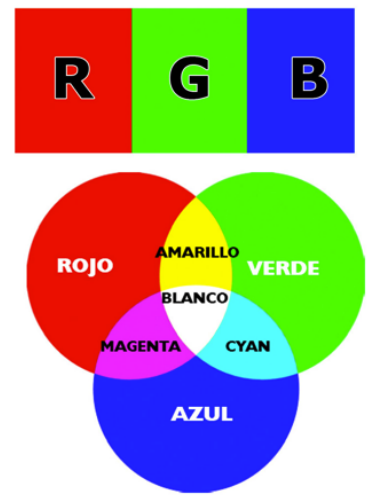

Figura 3 - Modelo de color RGB.

El modelo RGB asigna un valor numérico de 1 a 3 dígitos, que representa a cada uno de los colores primarios, el que a su vez determina la proporción en que es mezclado cada color, de manera que un valor "o" para cada valor de RGB significaría que éste no interviene en la mezcla y a medida que el valor aumenta, éste aporta más intensidad a la coloración. Así de manera usual, la intensidad de cada uno de los colores componentes, se mide según en una escala que va del o a 255 (Li et al., 2007; Sankaranarayanan \& Sivanantha, 2006).

La técnica de procesamiento de imagen utilizada, se basa en que la solución contenedora del ion específico tiene un color definido en función de la concentración de cobre que posea, de manera que ésta solución puede ser correlacionada con una determinada concentración del ion (en nuestro caso ion Cobre II) específico. La metodología consiste entonces en utilizar el color de la solución como un medio para medir, a través de un programa de tratamiento de imágenes, la intensidad de los colores primarios y basados en esta medición, encontrar un modelo matemático de estimación que permita correlacionar la imagen de color obtenida, con la concentración de cobre en la solución. 
A partir de las diversas imágenes capturadas para cada tipo de muestra desde el proceso, se aplica el procesamiento de imágenes que consiste en la descomposición de la imagen en sus canales Rojo, Verde y Azul (RGB). Como resultado, para cada imagen capturada a una frecuencia de muestreo definida, se obtienen tres matrices cuyas celdas son valores numéricos que varían entre o y 255 . Con posterioridad, para cada una de las matrices obtenidas se determina la media y este valor numérico escalar, es asignado a las variables R, G y B. Los valores escalares de R, G y B, posteriormente son utilizados en un modelo matemático que permite estimar, en tiempo real, la concentración de cobre para cada una de las muestras capturadas. Los parámetros de la ecuación que modela la correlación de los componentes R, G y B de cada muestra con su concentración de cobre, fueron obtenidos mediante la aplicación de Algoritmos Genéticos, cuya función objetivo fue la minimización del error cuadrático entre los valores de concentración de cobre determinados mediante ensayos de laboratorio y el valor estimado por el modelo de correlación. En la siguiente sección se presenta este procedimiento, de manera más detallada.

\section{Resultados experimentales}

Para comprobar la reproducibilidad de los datos obtenidos al realizar mediciones, se toman múltiples muestras con diferentes concentraciones en un intervalo comprendido entre $30 \mathrm{~g} / \mathrm{L} \mathrm{y} \mathrm{46,0} \mathrm{g/L} \mathrm{de} \mathrm{electrolito} \mathrm{rico} \mathrm{de} \mathrm{Cobre} \mathrm{(II).} \mathrm{Estas} \mathrm{muestras}$ son preparadas por dilución a partir de la muestra de mayor concentración, como muestra la Figura 4. El valor de concentración de estas muestras, fue determinada en un Laboratorio Químico de la Universidad de Antofagasta, mediante el método de la espectrofotometría.

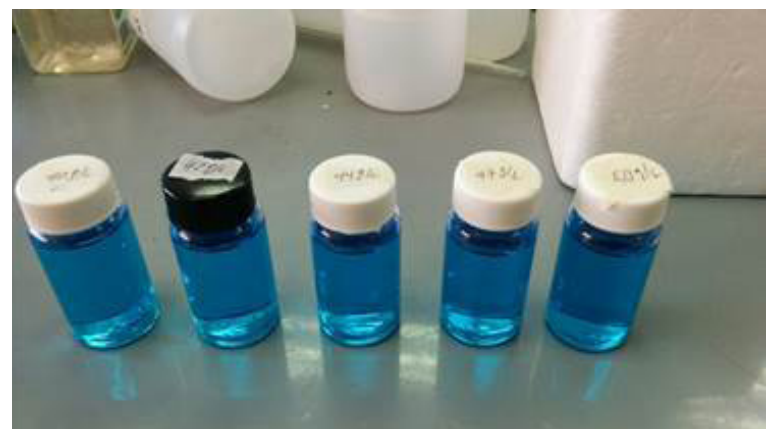

Figura 4 - Muestras obtenidas por dilución.

Las mediciones se realizan empezando con la muestra de menor concentración (se capturan diversas imágenes de cada muestra), para concluir con la muestra de mayor concentración, repitiendo la medición en varias ocasiones para observar qué comportamiento tiene la técnica en relación con los valores obtenidos y analizar la reproducibilidad de estas mediciones. Con los datos conseguidos, se genera la Tabla 1 (en esta tabla solamente se presenta solo un pequeño número de los experimentos realizados). 
Como se puede observar en la Tabla 1, los datos (escalares) de los tres grupos, R, G y B, mantienen valores muy cercanos para cada muestra de una determinada concentración preparada en laboratorio.

\begin{tabular}{|c|c|c|c|c|c|}
\hline Experimento & Muestra & Concentración [g/l] & $\boldsymbol{R}$ & $\boldsymbol{G}$ & $\boldsymbol{B}$ \\
\hline 1 & 1 & 30,00 & 20 & 121 & 158 \\
\hline 2 & 1 & 30,00 & 21 & 123 & 160 \\
\hline 3 & 1 & 30,00 & 21 & 121 & 158 \\
\hline 4 & 1 & 30,00 & 20 & 123 & 160 \\
\hline 1 & 2 & 35,00 & 18 & 118 & 158 \\
\hline 2 & 2 & 35,00 & 19 & 119 & 158 \\
\hline 3 & 2 & 35,00 & 18 & 118 & 158 \\
\hline 4 & 2 & 35,00 & 18 & 115 & 158 \\
\hline 1 & 3 & 41,30 & 17 & 117 & 161 \\
\hline 2 & 3 & 41,30 & 17 & 115 & 158 \\
\hline 3 & 3 & 41,30 & 17 & 116 & 160 \\
\hline 4 & 3 & 41,30 & 17 & 116 & 160 \\
\hline 1 & 4 & 42,20 & 16 & 116 & 161 \\
\hline 2 & 4 & 42,20 & 16 & 117 & 162 \\
\hline 3 & 4 & 42,20 & 16 & 116 & 160 \\
\hline 4 & 4 & 42,20 & 16 & 115 & 160 \\
\hline 1 & 5 & 43,10 & 15 & 115 & 162 \\
\hline 2 & 5 & 43,10 & 16 & 116 & 163 \\
\hline 3 & 5 & 43,10 & 15 & 113 & 159 \\
\hline 4 & 5 & 43,10 & 14 & 113 & 161 \\
\hline 1 & 6 & 44,50 & 14 & 112 & 161 \\
\hline 2 & 6 & 44,50 & 14 & 116 & 165 \\
\hline 3 & 6 & 44,50 & 14 & 116 & 166 \\
\hline 4 & 6 & 44,50 & 13 & 114 & 165 \\
\hline 1 & 7 & 46,00 & 13 & 110 & 166 \\
\hline 2 & 7 & 46,00 & 12 & 108 & 163 \\
\hline 3 & 7 & 46,00 & 12 & 108 & 163 \\
\hline 4 & 7 & 46,00 & 12 & 107 & 163 \\
\hline
\end{tabular}

Tabla 1 - Resultado de cuatro mediciones por cada muestra.

Como se mencionó en la sección anterior, haciendo un proceso de ajuste de datos basado en los resultados experimentales, se sigue un procedimiento para obtener un modelo que represente la concentración de cobre en función de los valores RGB.

Para trabajar con los modelos de estimación se utilizan: la ecuación de segundo grado 1.1 y la ecuación de primer grado 1.2. 


$$
\begin{gathered}
\text { Conc }=A_{1}{ }^{*} \mathrm{Fcol}^{2}+A_{2} * \mathrm{Fcol}+A_{3} \\
\text { Fcol }=C_{1}{ }^{*} R+C_{2} * G+C_{3} * B
\end{gathered}
$$

Se tiene de esta manera un modelo que tiene 6 coeficientes que deben ser determinados. La determinación de estos coeficientes se realiza mediante un algoritmo de optimización, el cual minimiza el error cuadrático entre el valor de concentración medido y la concentración estimada mediante las ecuaciones (1.1) y (1.2). El modelo de optimización se presenta en (1.3).

$$
\min x \sum_{k}^{35}\left(C O N_{K}^{\text {med }}-\operatorname{CON}_{k}^{\text {estimada }}(x)\right)^{2}
$$

con $x^{\min } \leq x \leq x^{\max } ; C O N_{k}^{\text {med }}$ corresponde a la concentración medida (en laboratorio) en el experimento $\mathrm{k}, C O N ?_{k}^{\text {estimada }}$ es la concentración estimada para el experimento $\mathrm{k}$ mediante las ecuaciones (1.1) y (1.2). El vector de parámetros de diseño queda definido por $x=\left[C_{1} C_{2} C_{3} A_{1} A_{2} A_{3}\right]$.

Para resolver el problema de modelamiento se utilizaron y compararon diferentes técnicas clásicas de identificación, habiéndose elegido finalmente la técnica de Algoritmos Genéticos (GA) que entregó un menor error de ajuste.

Una vez determinados los parámetros de diseño, la ecuación (1.1) entregará la concentración estimada y representa el modelo matemático que permite asociar la tonalidad que exhibe la muestra con su concentración de cobre, a través de los coeficientes $A_{\mathrm{i}}$ y Fcol (Factor color). La ecuación (1.2) determina el Fcol en función de los valores RGB obtenidos en el procesamiento de la imagen.

Para trabajar con los modelos propuestos, se usan los datos proporcionados por la Tabla 1, datos que fueron procesados mediante el Toolbox de Algoritmos Genéticos de Matlab, para generar los coeficientes $\mathrm{C}_{1}, \mathrm{C}_{2}, \mathrm{C}_{3}, \mathrm{~A}_{1,} \mathrm{~A}_{2 \mathrm{y}} \mathrm{A}_{3}$, que son mostrados en Tabla 2.

\begin{tabular}{lll}
\hline C1 & C2 & C3 \\
\hline $\boldsymbol{o . 9 4 8 9 3 8 5 8}$ & 0.21013730 & 0.33678871 \\
\hline A1 & A2 & A3 \\
\hline -o.09042402 & -3.89993823 & 3.99293590 \\
\hline
\end{tabular}

Tabla 2 - Coeficientes $\mathrm{C}_{1}, \mathrm{C}_{2}, \mathrm{C}_{3}, \mathrm{~A}_{1}, \mathrm{~A}_{2} \mathrm{y} \mathrm{A}_{3}$

Para el proceso de validación del modelo, se utilizan los coeficientes de la Tabla 2 y las ecuaciones (1.1) y (1.2). Los resultados se consignan en la Tabla 3, en la cual se registra el Fcol, la concentración estimada y el cálculo del error al cuadrado (SE) entre la concentración de la muestra y la concentración arrojada por el modelo. Con estos valores de SE, se determina el Error Cuadrático Medio, MSE, y la Raíz del Error Cuadrático Medio o RMSE, valores que se registran en la Tabla 4. 


\begin{tabular}{|c|c|c|c|c|}
\hline Muestra & Conc. muestra $[g / l]$ & Fcol & Conc. Modelo [g/l] & $S E$ \\
\hline 1 & 30,00 & -8.807231 & 31.33 & 1.76 \\
\hline 1 & 30,00 & -8.133654 & 29.73 & 0.07 \\
\hline 1 & 30,00 & -8.111595 & 29.68 & 0.10 \\
\hline 1 & 30,00 & -8.723745 & 31.13 & 1.28 \\
\hline 2 & 35.00 & -11.335520 & 36.58 & 2.50 \\
\hline 2 & 35.00 & -11.335520 & 36.58 & 2.50 \\
\hline 2 & 35.00 & -11.672309 & 37.19 & 4.82 \\
\hline 2 & 35.00 & -11.965932 & 37.71 & $7 \cdot 36$ \\
\hline 3 & 41,30 & -13.841751 & 40.65 & 0.42 \\
\hline 3 & 41,30 & -13.588448 & 40.29 & 1.02 \\
\hline 3 & 41,30 & $-13 \cdot 378311$ & 39.98 & 1.73 \\
\hline 3 & 41,30 & -13.715100 & 40.47 & 0.69 \\
\hline 4 & 42,20 & -15.127478 & 42.30 & 0.01 \\
\hline 4 & 42,2 & -13.653672 & 40.38 & $3 \cdot 30$ \\
\hline 4 & 42,2 & -14.453901 & 41.47 & 0.53 \\
\hline 4 & 42,20 & -15.210964 & 42.39 & 0.04 \\
\hline 5 & 43,10 & -15.486325 & 42.70 & 0.16 \\
\hline 5 & 43,10 & $-15 \cdot 337616$ & 42.54 & 0.32 \\
\hline 5 & 43,10 & -15.906600 & 43.15 & 0.00 \\
\hline 5 & 43,10 & -16.518750 & 43.74 & 0.41 \\
\hline 6 & 44,50 & -18.749619 & $45 \cdot 33$ & 0.68 \\
\hline 6 & 44,50 & -17.572281 & 44.60 & 0.01 \\
\hline 6 & 44,50 & -16.898704 & 44.07 & 0.18 \\
\hline 6 & 44,50 & -17.235493 & 44.35 & 0.02 \\
\hline 7 & 46,00 & -19.782044 & $45 \cdot 76$ & 0.06 \\
\hline 7 & 46,00 & -21.973544 & 46.03 & 0.00 \\
\hline 7 & 46,00 & -21.151257 & 46.03 & 0.00 \\
\hline 7 & 46,00 & -20.814468 & 45.99 & 0.00 \\
\hline
\end{tabular}

Tabla 3 - Resultado de concentraciones y SE determinadas a partir del modelo.

\begin{tabular}{ll}
\hline Indicador & Valor \\
\hline MSE & 1.18 \\
\hline RMSE & 1.09 \\
\hline
\end{tabular}

Tabla 4 - Indicadores estadísticos de los datos experimentales.

Finalmente, se configura el modelo en la unidad de procesamiento de imágenes y se realizan las lecturas para las concentraciones de cada una de las muestras. 
La Tabla 5 registra las concentraciones de las muestras, el valor de concentración entregado por el analizador, correspondiente a cada muestra, y el valor del Fcol correspondiente.

\begin{tabular}{lll}
\hline Concentración muestra $[\boldsymbol{g} / \mathbf{l}]$ & Fcol & Concentración medida $[\boldsymbol{g} / \mathbf{l}]$ \\
\hline $\mathbf{3 0 . 0 o}$ & -8.192188 & 29.73 \\
\hline $\mathbf{3 5 . 0 0}$ & -11.364503 & 36.58 \\
\hline $\mathbf{4 1 . 3 0}$ & -13.538357 & 40.29 \\
\hline $\mathbf{4 2 . 2 0}$ & -14.554653 & 41.47 \\
\hline $\mathbf{4 3 . 1 0}$ & -15.949196 & 43.15 \\
\hline $\mathbf{4 4 . 5 0}$ & -17.812161 & 44.60 \\
\hline $\mathbf{4 6 . 0 0}$ & -20.949184 & 45.99 \\
\hline
\end{tabular}

Tabla 5 - Concentración de la muestra, Fcol y Concentración medida en g/l.

La Figura 5 muestra un gráfico de los valores registrados en la Tabla 5. Se observa un buen ajuste del modelo a los valores de las muestras, comportamiento esperado de acuerdo a los indicadores determinados previamente, MSE y RMSE.

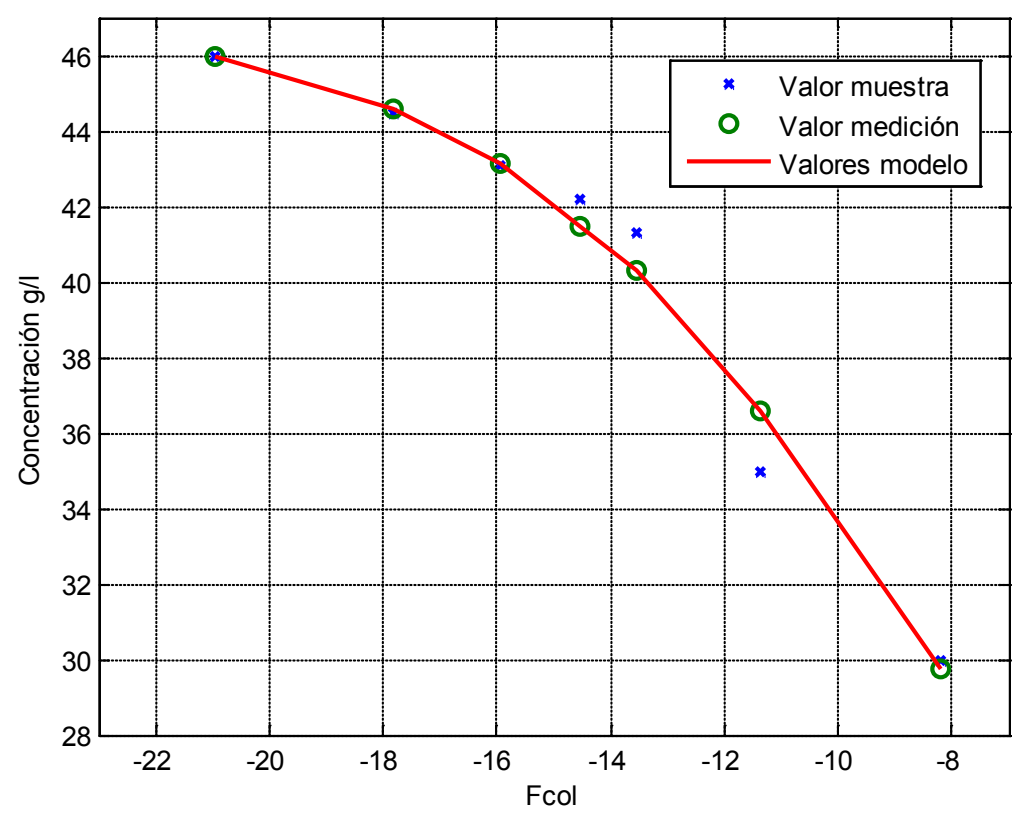

Figura 5 - Factor Color versus Concentración muestra, Concentración medida y Concentración modelo. 


\subsection{Equipos y programas utilizados para configurar el Analizador}

- Computador con procesador Intel Core i3, 8 Gb RAM DDR,

- Tarjeta de adquisición de vídeo 3DEMON de 8MB,

- Cámara de vídeo, modelo SONY CCD-TRV34,

- Plataforma .NET: procesamiento de las imágenes,

- PLC RSLogic 1100: automatización del proceso de medición.

\section{Conclusiones y trabajo futuro}

De acuerdo a los resultados experimentales, se demuestra que existe una relación importante entre las concentraciones de soluciones acuosas y la combinación de los colores primarios del modelo RGB, en el rango de concentraciones estudiados, lo que motiva a seguir per feccionando el analizador desarrollado. Se debe considerar además que por ser este instrumento un prototipo, fue implementado con componentes y recursos constructivos de muy bajo coste, por ejemplo, se utilizó una cámara del tipo webcam para la captura de imágenes, se puede esperar que la precisión de las mediciones mejore, utilizando componentes de una mejor calidad.

La relevancia de poder contar con un analizador de concentraciones en línea, y que sea de bajo costo, es alta para la operación y control del proceso de SX, siendo ésta su principal variable operacional.

Un aspecto importante a considerar para el perfeccionamiento del analizador, es que los cambios en tipo y cantidad de los elementos que componen las soluciones acuosas, en las diferentes plantas de SX, puede requerir compensaciones en el sensor para mantener su precisión. Una forma de compensar es recalibrando el equipo para cada planta de SX a cada cierto período, parámetro que se determinará en las pruebas a realizar en plantas industriales.

Como trabajo futuro, se propone sustituir el modelo de estimación representado por la ecuación 1.1, por una red neuronal de aprendizaje supervisado, que permita a partir de los componentes RGB de las imágenes capturadas, estimar la concentración de cobre que posee el acuoso, sustituyendo además el computador por una tarjeta embebida para el procesamiento de la red neuronal ya entrenada.

Finalmente, se debe destacar que esta innovación se encuentra en proceso de evaluación de la protección industrial en INAPI (Chile), con número de solicitud 2665-2016 (prioridad), con fecha 20/10/2016.

\section{Referencias}

Artigas, J. M., Capilla P., Malo J., Martínez F., Luque M., \& Pujol J., (2002), Fundamentos de colorimetría, Valencia, España: Universitat de València.

Bergha, L.G., Jämsä-Jounela, S.L., \& Hodouinc, D. (2001). State of the art in copper hydrometallurgic processes control Bergha. Control Engineering Practice 9, 1007-1012.

Komulainen, T., Doyle III, F., Rantala, A., \& Jämsä-Jounela, S. L. (2009). Control of an industrial copper solvent extraction process. Journal of Process Control, 19, 2-15. 
Li, G., Yuan, R., Yang, Z. \& Huang, X. (2007). A yellow license plate location method based on RGB model of color image and texture of plate. In Digital Media and its Application in Museum \& Heritages, Second Workshop on (pp. 42-46). IEEE.

Martínez, B., Gil Novoa, O. D., \& Barón González, A. J. (2006). Espectrometría de Fluorescencia de Rayos X. Revista Colombiana de Física, 38(2), 790-793.

Mwale, M. \& Megaw, D. (2011). Development of effective solvent-extration process control - low cost implementation value-addition to hydrometallurgical copper operations. In Proceedings Sixth Southern African Base Metals Conference. Southern African Institute of Mining \& Metallurgy, 353-365, Johannesburg.

Sankaranarayanan, K., Sivanantha Raja, A., (2006). Use of RGB Color Sensor in Colorimeter for better Clinical measurement of Blood Glucose. BIME Journal, 6(1), $23-28$. 